\title{
Essential facets of competence that enable trust in medical graduates: a ranking study among physician educators in two countries
}

\author{
Marjo Wijnen-Meijer • Marieke van der Schaaf • \\ Kirstin Nillesen · Sigrid Harendza • Olle ten Cate
}

Published online: 19 October 2013

(C) The Author(s) 2013. This article is published with open access at Springerlink.com

\begin{abstract}
One way to operationalize the assessment of trainees in a competency-based context is to determine whether they can be entrusted with critical activities. To determine which facets of competence (FOCs) are most informative for such decisions, we performed a Delphi study among Dutch educators. In the current study, the resulting list of facets of competence was evaluated among experienced Dutch and German clinical educators to determine which facets appear most relevant and to evaluate the agreement among experts in different countries as a support for their external validity. Eight Dutch and eight German experts scored each FOC on a five-point scale for relevance. A rank-order comparison showed that there was almost full agreement about the top 10 FOCs, among which 'Scientific and empirical grounded method of working', 'Knowing and maintaining own personal bounds and possibilities', 'Active professional development', 'Teamwork and collegiality', 'Active listening to patients', and 'Verbal communication with colleagues and supervisors'. We conclude that these facets of competence may be used in a training for educators who need to make entrustment decisions about trainees.
\end{abstract}

Keywords Competences $\cdot$ Entrustability $\cdot$ Assessment $\cdot$ Supervisors' perceptions

\footnotetext{
M. Wijnen-Meijer $(\varangle) \cdot$ K. Nillesen $\cdot$ O. ten Cate

Center for Research and Development of Education, University Medical Center Utrecht, PO Box 85500, 3508 GA Utrecht, the Netherlands

email: m.wijnen-meijer@umcutrecht.nl

M. van der Schaaf

Department of Education, Utrecht University, Utrecht, the Netherlands

S. Harendza

Department of Internal Medicine, University Medical Center Hamburg-Eppendorf, Hamburg, Germany
} 


\section{Introduction}

Following a rapid increase in the popularity of competency-based medical education $[1,2]$, the methods and concerns around the assessment of competence have been met with increasing interest [3, 4]. Assessment tools in a workplace that cannot be standardized and increased interest in the 'softer' skills pose challenges to the assessment procedures $[5,6]$. One approach that has been suggested to operationalize the attainment of competencies is to determine whether or when a trainee can be trusted to execute a professional activity without supervision [7]. Trust in trainees requires observations that do not only draw on standardized skills and knowledge but take other facets of competence into account [8,9]. Using a Delphi approach, we investigated the factors that educators in the Netherlands find important to consider when making entrustment decisions about medical trainees [10]. This yielded a list of 25 relevant factors or 'facets of competence' (FOCs) when entrusting trainees with clinical responsibilities.

The aim of the current study was to determine the external validity, i.e. the generalizability, of the factors that Dutch educators found essential. Our approach was to ask experienced educators in two countries to rank-order these FOCs and then to determine the level of agreement among the countries about the highest scoring FOCs.

The study was carried out among Dutch and German medical educators. The Netherlands and Germany differ in medical education culture, particularly in the sense that in the Netherlands education reform has dominated medical curricula throughout the country since the mid-1970s, while such processes in Germany have started only recently $[11,12]$. It is fair to say that Dutch medical schools have 'modern medical curricula' (not necessarily 'better') and most German medical schools have predominantly 'traditional medical curricula', while a number of other countries in Europe have positions in between. Agreement among medical educators in the Netherlands and Germany about important FOCs would support the generalizability of those FOCs.

\section{Methods}

\section{Participants}

To find comparable groups in both countries we approached Dutch and German experts. We approached all 24 experienced clinicians in the Netherlands who met the following criteria: (1) holds an academic chair in medical education, (2) works in clinical practice and (3) supervises residents. The 36 German physicians we approached met the following criteria: (1) Master's degree in medical education, (2) works in clinical practice and (3) supervises residents.

\section{Questionnaire}

The experts were invited by email to complete a questionnaire in an electronic format, after the German version had been translated from the Dutch language. To gain insight into the experts' judgements about FOCs, they were asked to assign a 
score from 1 ('least important') to 5 ('most important') to each of the 25 FOCs that had resulted from the Delphi study. They were requested to give each possible score (1-5) five times (so, 5 FOCs had to get score 1; 5 FOCs had to get score 2, etc.).

Data analysis

We calculated means, medians and standard deviations for both the Dutch and German group of experts and a level of agreement, according to an adapted De Loe's [13] procedure. De Loe developed a method to determine the amount of consensus, based on the percentages of answers in one or two contiguous categories of a rating scale (see Table 1). De Loe used this method for a 4-point scale, while we used the same method for a 5-point scale, which makes it a little more stringent.

\section{Results}

Respondents

In total $8 / 24$ Dutch and $8 / 36$ German experts participated in the ranking study (response rates 33 and $22 \%$, respectively). In the Dutch group, 7 were male and the average age was 61 years (56-66 years). In the German group, the average age was 43 years (33-53 years) with 4 male and 4 female responders. The responders in both rounds represented a wide range of surgical and non-surgical disciplines (Dutch: cardiology, general practice, gynaecology, internal medicine, neurology, oncology and surgery; German: emergency medicine, gynaecology, internal medicine, psychiatry and surgery).

\section{Results}

The results of the ranking study are presented in Table 2. The medians and means indicate the ranking of the FOCs. For the Dutch group of responders, medians were between 1 and 5 and the means between 1.63 and 4.75 (SD 0.46-1.64). For the German group, medians varied between 1 and 5 and the means between 1.88 and 4.38 (SD 0.54-1.93). For both groups, the level of agreement varied from none to high.

Table 1 Levels of agreement according to De Loe [13]

\begin{tabular}{llc}
\hline Agreement & \multicolumn{2}{l}{ Calculation level of agreement 4-point scale according to De Loe } \\
\cline { 2 - 3 } & 1 category & 2 contiguous categories \\
\hline High (\%) & 70 & 80 \\
Medium (\%) & 60 & 70 \\
Low (\%) & 50 & 60 \\
None & $<60 \%$ of ratings in 2 contiguous categories \\
\hline
\end{tabular}




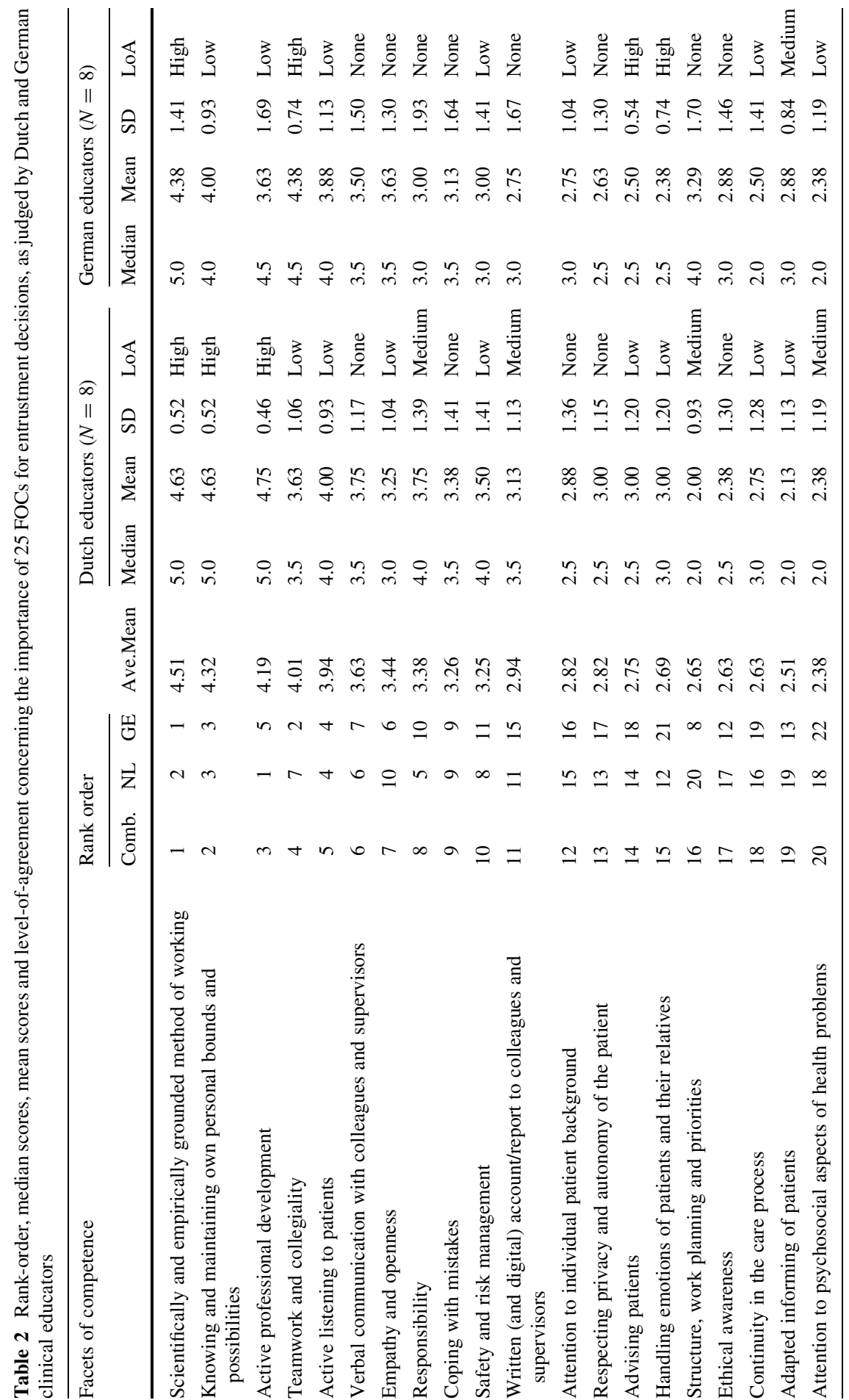




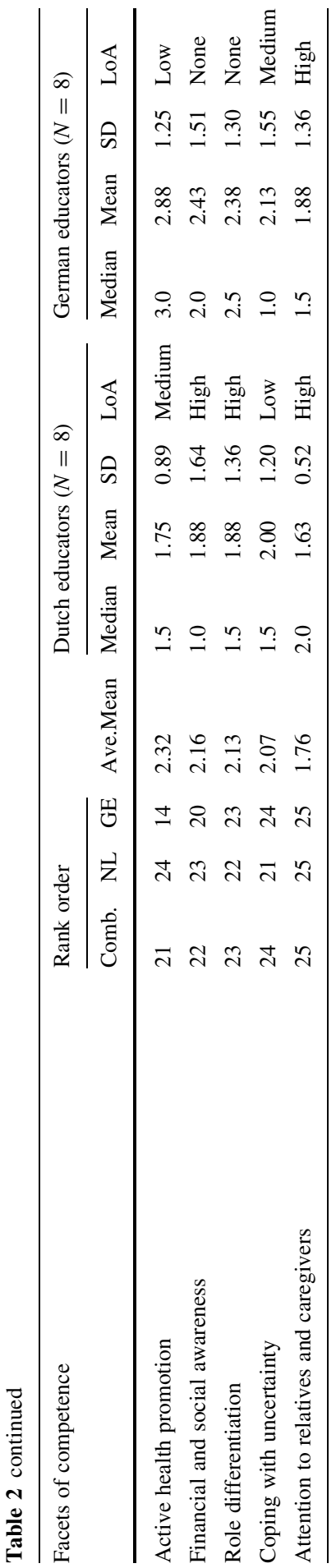




\section{Discussion}

The aim of this study was to determine which FOCs of medical trainees are considered most important to formulate entrustment decisions by experienced supervisors in residency training and to evaluate the agreement about them in different European countries, to expand their generalizability.

We found strong agreement between physician educators from the Netherlands and physician educators from Germany in ranking the competency facets relevant for entrustment decisions. The 'top 10' for the total group of responders, based on medians and means, appeared nearly the same as the 'top 10' for each of the two countries separately. The only FOC that substantially differed was 'structure, work planning and priorities', ranked 8 in the German group, but 20 among the Dutch.

The fact that two groups of physician educators from different medical education cultures highly agreed on the importance of certain FOCs for entrustment decisions strengthens the relevance of these FOCs. The top-10 align with Kennedy et al. [9]'s findings. In their grounded theory study, these authors also found 'truthfulness' (the absence of deception) and 'conscientiousness' (the thoroughness in data gathering and dependability) to be an important quality that supervisors value in trainees to determine their readiness for independent clinical work [9]. We had not explicitly included these as facets in our list, but it may be assumed that our respondents would score them highly if they had been included. Implicitly, they are reflected in our items that stress scientifically grounded working, openness, responsibility and coping with mistakes, all part of the top-10.

One limitation of our study is the low response, which also varied in the different parts of the study. However, the expertise of the participants and the fact that a wide range of disciplines are included adds to the relevance and the generalized nature of the findings, but the study cannot be viewed as conclusive. Another limitation is that we only included one other country. Future studies to establish a generalized nature of factors that affect entrustment decisions should include countries with other than the Western industrialized culture, as we cannot exclude that those countries would show different factors.

The background of making entrustment decisions deserves further empirical study, preferably with more experts and more countries and focused on a validation in practice.

As a practical outcome, our findings may be used as input for a frame of reference training [14] for clinicians who must regularly take entrustment decisions. Supervisors may be guided by their first impressions of trainees, which may be less accurate than they tend to think [15], and training can make them aware to take multiple facets of competence into account.

\section{Conclusion}

Our aim was to reveal what facets of competence are considered most important for entrustment decisions by supervisors of residents. We found high consensus between experts from the Netherlands and Germany, despite large differences in their 
curricula. Our findings are relevant for the development of assessment instruments to evaluate whether medical graduates are ready for clinical practice.

\section{Essentials}

- There is high agreement among supervisors about what facets of competence are considered most important for entrustment decisions.

- There is consensus between physician educators from two countries with different medical education climates (the Netherlands and Germany).

Open Access This article is distributed under the terms of the Creative Commons Attribution License which permits any use, distribution, and reproduction in any medium, provided the original author(s) and the source are credited.

\section{References}

1. Frank JR, Snell LS, ten Cate O, et al. Competency-based medical education: theory to practice. Med Teach. 2010;32(8):638-45.

2. Carraccio C, Wolfsthal SD, Englander R, Ferentz K, Martin C. Shifting paradigms: from Flexner to competencies. Acad Med. 2002;77(5):361-7.

3. Norcini JJ, Holmboe ES, Hawkins RE. Evaluation challenges in the era of outcomes-based education. In: Holmboe ES, Hawkins RE, editors. Practical guide to the evaluation of clinical competence. 1st ed. Philadelphia: Mosby Elsevier; 2008. p. 1-9.

4. Lurie SJ. History and practice of competency-based assessment. Med Educ. 2012;46(1):49-57.

5. Lurie SJ, Mooney CJ, Lyness JM. Commentary: pitfalls in assessment of competency-based educational objectives. Acad Med. 2011;86(4):412-4.

6. Lurie SJ, Mooney CJ, Lyness JM. Measurement of the general competencies of the accreditation council for graduate medical education: a systematic review. Acad Med. 2009;84(3):301-9.

7. Ten Cate O. Entrustability of professional activities and competency-based training. Med Educ. 2005;39(12):1176-7.

8. Sterkenburg A, Barach P, Kalkman C, Gielen M, ten Cate O. When do supervising physicians decide to entrust residents with unsupervised tasks? Acad Med. 2010;85(9):1408-17.

9. Kennedy TJT, Regehr G, Baker GR, Lingard L. Point-of-care assessment of medical trainee competence for independent clinical work. Acad Med. 2008;83(10 Suppl):S89-92.

10. Wijnen-Meijer M, Van der Schaaf M, Nillesen K, Harendza S, ten Cate O. Essential facets of competence that enable trust in graduates: a Delphi study among physician educators in the Netherlands. J Grad Med Educ. 2013;5:46-53.

11. Ten Cate O. Medical education in the Netherlands. Med Teach. 2007;29(8):752-7.

12. Nikendei C, Weyrich P, Ju J, Schrauth M. Medical education in Germany. Med Teach. 2009;31:591-600.

13. De Loe RC. Exploring complex policy questions using policy Delphi. A multi-round, interactive survey method. Appl Geogr. 1995;15(1):53-68.

14. Holmboe ES, Ward DS, Reznick RK, et al. Faculty development in assessment: the missing link in competency-based medical education. Acad Med. 2011;86(4):460-7.

15. Wood TJ. Exploring the role of first impressions in rater-based assessments. Adv Health Sci Educ. 2013;. doi:10.1007/s10459-013-9453-9.

\section{Author Biographies}

Marjo Wijnen-Meijer is assistant professor in Medical Education at the Center for Research and Development of Education at UMC Utrecht, the Netherlands. 
Marieke van der Schaaf is associate professor and coordinator in Master Educational Design and Consultancy at the Department of Education, Utrecht University, the Netherlands.

Kirstin Nillesen is educationalist and worked as a trainee at the Center for Research and Development of Education at UMC Utrecht, the Netherlands.

Sigrid Harendza is professor of internal medicine and educational research at the Department of Internal Medicine, University Medical Center Hamburg-Eppendorf, Germany.

Olle ten Cate is professor of Medical Education at UMC Utrecht and director of the Center for Research and Development of Education at UMC Utrecht, the Netherlands. 